\title{
¿Se observarán cambios en el consumo de alcohol y tabaco durante el confinamiento por COVID-19?
}

\section{Will changes in alcohol and tobacco use be seen during the COVID-19 lockdown?}

\author{
Leticia García-Álvarez *******,****,*****, Lorena de la Fuente-Tomás*,******,***, Pilar \\ Alejandra Sáiz ***,***,****,******, Ma Paz García-Portilla*,**,***,****,******, Julio \\ BobES $* * *, * * *, * * * *, * * * * * *$ \\ * Instituto de Investigación Sanitaria del Principado de Asturias (ISPA), España \\ ** Área de Psiquiatría. Universidad de Oviedo, España \\ *** Centro de Investigación Biomédica en Red de Salud Mental. CIBERSAM, España \\ **** Instituto de Neurociencias del Principado de Asturias (INEUROPA), España \\ ***** Departamento de Psicología. Universidad de Oviedo, España \\ ****** Servicio de Salud del Principado de Asturias. SESPA, España
}

\section{Introducción}

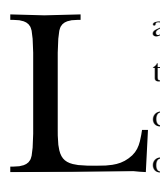
a pandemia por coronavirus (COVID-19) registrada en los últimos meses ha causado una situación de emergencia a nivel mundial. Se estima que las reacciones de ansiedad, preocupación o miedo sean frecuentes en la sociedad dado su carácter desconocido y novedoso junto con las medidas de distanciamiento social derivadas del estado de alarma. Sin embargo, todavía se desconoce el impacto psicológico que puede tener no solo el coronavirus per se, sino el confinamiento, ya que nos encontramos ante una situación excepcional sin precedentes.

Algunos estudios realizados sobre el impacto del síndrome respiratorio agudo grave (SARS, por sus siglas en inglés Severe Acute Respiratory Syndrome), el primer brote masivo de una enfermedad infecciosa en el siglo XXI, han mostrado un impacto significativo sobre la salud mental de las personas y su nivel de bienestar (Ko, Yen, Yen y Yang, 2006), incluso 4 años después de la epidemia (Lam et al., 2009). Se habla de "bio-desastres" capaces de generar un impacto psicológico comparable al de otras catástrofes como ata- ques terroristas, terremotos, etc. (Chong et al., 2004; Wu et al., 2008). En el caso de las personas expuestas al SARS se ha mostrado cómo el trastorno de estrés postraumático y los trastornos depresivos han sido las alteraciones psicológicas más prevalentes durante el seguimiento a largo plazo (Mak, Chu, Pan, Yiu y Chan, 2009). Sin embargo, durante el SARS tanto las medidas llevadas a cabo como el nivel de afectación global no fueron tan extremos como en esta ocasión con lo que sería de esperar que el impacto de esta pandemia por COVID-19 sea todavía mayor.

Durante el brote de síndrome respiratorio de coronavirus de Oriente Medio (MERS-CoV, por sus siglas en inglés Middle East Respiratory Syndrome Coronavirus) acaecido en 2015 y que generó el confinamiento de casi 17.000 personas expuestas al mismo, se observó un mayor riesgo de síntomas de estrés postraumático en sanitarios que habían tratado a pacientes infectados (Lee, Kang, Cho, Kim y Park, 2018), así como síntomas de ansiedad (7,6\%), ira $(16,6 \%)$ y depresión $(19,3 \%)$, incluso entre aquellos que sufrieron medidas de aislamiento sin haber desarrollado la enfermedad (Yoon, Kim, Ko y Lee, 2016), perdurando, en 
muchos casos, durante los 4-6 meses posteriores al confinamiento (Jeong et al., 2016).

En los estudios publicados sobre el impacto psicológico del COVID-19 en China, se ha observado malestar emocional, estando las respuestas de ansiedad grave presentes en un tercio de la población general (Lima et al., 2020; Wang et al., 2020). Sin embargo, en un estudio llevado a cabo con una amplia muestra de población general española, se ha observado cómo durante las primeras semanas de confinamiento (del 19 al 26 de marzo) las respuestas emocionales desadaptativas observadas, de modo más frecuente, han sido los síntomas depresivos (46,7\%), seguidos por un estilo evitativo de afrontamiento $(44,3 \%)$. Además, en contra de lo esperado, las respuestas de ansiedad fueron las menos frecuentes, afectando al 6,1\% de la población (García-Álvarez et al., 2020). Asimismo, los efectos psicológicos del confinamiento parecen incrementarse con el paso de días (GarcíaÁlvarez et al., 2020) y en ciertos grupos vulnerables de la población, tales como el personal sanitario (Bai et al., 2004; Maunder et al., 2003), personas con enfermedades somáticas previas o personas con un trastorno mental previo o actual, más concretamente, depresión, ansiedad o trastorno bipolar (García-Álvarez et al., 2020). De igual forma, aquellas personas con problemas por consumo de sustancias podrían ser colectivos en riesgo (Pfefferbaum y North, 2020).

Se plantea cómo esta situación de emergencia en la salud pública podría generar no sólo reacciones emocionales negativas, sino también la falta de cumplimiento con las directivas de la salud pública y la génesis de comportamientos poco saludables como el uso excesivo de sustancias (Pfefferbaum y North, 2020). Entre las respuestas más frecuentes del confinamiento se ha planteado que podrían estar: estrés, depresión, irritabilidad, insomnio, miedo, confusión, enfado, frustración, aburrimiento o estigma, y además, existe una preocupación acerca de que esos síntomas puedan persistir tiempo después de ese período de cuarentena (Brooks et al., 2020). Además, también se ha señalado que tales medidas podrían tener un impacto notable sobre el incremento de riesgo suicida en la población (Reger, Stanley y Joiner, 2020).

Ante un cambio tan radical en nuestro comportamiento y costumbres (distanciamiento social, teletrabajo, limitación en la realización de actividades deportivas y de ocio fuera de nuestras casas, etc.), no cabe sino la búsqueda de actividades alternativas. Se habla de la importancia de la expresión de emociones negativas, mantenerse el contacto con los familiares y amigos, realizar actividades de manera regular, actividades de ocio, etc. (Park y Park, 2020). Sin embargo, otras actividades como el consumo de alcohol, tabaco y otras sustancias podrían incrementarse no solo como una forma de distracción o estrategia de evitación conductual, sino también como consecuencia del estrés, ansiedad o los síntomas depresivos que se están experimentando.

\section{Alcohol}

La exposición a situaciones potencialmente generadoras de trastornos de estrés postraumático como los ataques terroristas, desastres naturales (terremotos, erupciones volcánicas) o accidentes se ha asociado con un aumento en las tasas de abuso y dependencia de alcohol en algunos estudios (Boscarino, Adams y Galea, 2006; Lebeaut, Tran y Vujanovic, 2020), aunque en otros, los resultados han ido en la dirección opuesta (North, Kawasaki, Spitznagel y Hong, 2004; Shimizu et al., 2000). Por ello, es fundamental analizar cuáles son los factores que pueden determinar las diferencias encontradas en estos estudios y ver hasta qué punto esos comportamientos pueden estar aumentando durante este período de confinamiento.

En el caso del SARS, el abuso o dependencia de alcohol se ha asociado con haber estado trabajando como personal sanitario durante la epidemia incluso tres años después del brote (Wu et al., 2008). Se identificaron como factores de riesgo un mayor grado de exposición al virus y haber tenido que ser aislado como consecuencia del mismo. Sin embargo, tener familiares afectados o fallecidos por el SARS o la exposición a noticias sobre la epidemia no se relacionaron con el abuso y dependencia de alcohol. Además, se identificó una relación dosis-respuesta entre la intensidad de la exposición al virus y los síntomas de abuso y dependencia de alcohol a largo plazo (Wu et al., 2008).

En relación a la pandemia por COVID-19, todavía no se disponen de datos de trastornos por abuso de sustancias ni estudios que evalúen el posible aumento del consumo como consecuencia del confinamiento.

\section{Tabaco}

El consumo de alcohol, tabaco y marihuana a menudo se produce de manera conjunta (Degenhardt, Hall y Lynskey, 2001). Se ha visto que el consumo de tabaco aumenta ante estresores ambientales de diversa índole, como los conflictos armados, desastres naturales, etc. Sin embargo, al igual que en el caso del alcohol (Sánchez-Autet et al., 2018), se ha planteado la posibilidad de que ese consumo esté mediado por síntomas depresivos o trastornos de estrés postraumático (Ben-Zur y Zeidner, 2009; Jiménez-Treviño et al., 2019; Gross, Bastian, Smith, Harpaz-Rotem y Hoff, 2020). También se han observado diferencias en función del género, concretamente parece que las mujeres recurren al consumo de tabaco para regular el afecto negativo con mayor frecuencia que los hombres (Japuntich et al., 2016).

En cuanto a la pandemia por COVID-19, las personas que fuman o utilizan vapeadores han sido identificadas como un colectivo más vulnerable a la infección y a las complicaciones asociadas a la misma (Cai, 2020), ya que patologías tales como las enfermedades cardiovaculares, o respiratorias comórbidas, más prevalentes en fumadores 
crónicos, se han asociado con un peor pronóstico en pacientes infectados por COVID-19 (Volkow, 2020). Por tanto, se debe mostrar especial atención sobre este colectivo dado su mayor riesgo de infección y consecuencias graves.

Por otro lado, también es necesario conocer hasta qué punto se está produciendo un cambio en el patrón de consumo de tabaco (nuevos consumidores, aumento de la frecuencia, intensidad del consumo, etc.) como consecuencia de las respuestas emocionales o como estrategia de evitación o alternativa al aburrimiento al confinamiento y la pandemia.

\section{Evaluación e intervención}

Por todo lo anteriormente expuesto, se hace necesaria una evaluación de las alteraciones emocionales y comportamentales no solo en el momento actual, sino también de tipo prospectivo, sobre todo en las personas en las que persistan dichas respuestas desadaptativas con el objetivo de crear intervenciones específicas y adaptadas a las necesidades actuales. Para ello es necesario conocer cuáles son los factores de vulnerabilidad, así como los factores protectores y diseñar programas adaptados.

Entre las medidas de apoyo psicológico que se han propuesto en China para abordar el COVID-19 se encuentra un enfoque multidisciplinar que incluya entre otros a psicólogos, psiquiatras y personal de enfermería especialista en salud mental; proporcionar información fiable y actualizada sobre la pandemia y establecer diferentes servicios para proporcionar apoyo psicológico, entre los que se puede incluir programas de tratamiento a través de internet (Xiang et al., 2020).

En España, además del abordaje habitual desde salud mental, que en estos momentos se está llevando a cabo, siempre que es posible, de manera telefónica, existen líneas de teléfono atendidas por psicólogos que prestan apoyo a los profesionales sanitarios, a los familiares de personas que se encuentran hospitalizados por COVID-19 o en los casos que ha tenido lugar un fallecimiento (informando a los familiares, pidiendo consentimiento para la sedación, facilitando un último contacto e informando de la muerte cuanto se produce) (Arango, 2020).

\section{Conclusiones}

La pandemia por coronavirus (COVID-19) y el confinamiento podría generar respuestas emocionales desadaptativas como estrés o depresión, pero también podría incrementar los comportamientos poco saludables como el uso excesivo de alcohol o tabaco.

Es necesario desarrollar medidas de apoyo psicológico para toda la población y, especialmente, para los colectivos más vulnerables, así como para aquellos que desarrollen trastornos como consecuencia del mismo.
Por otro lado, se deberá de tener en cuenta si las personas sometidas a situaciones de estrés y aislamiento, como el escenario que nos ha tocado vivir, recurren con mayor frecuencia al consumo de sustancias para aliviar las emociones negativas. Además, aquellas personas con un trastorno por abuso de sustancias en periodo de remisión, tendrán que enfrentarse a las tensiones y al deseo por consumir de forma más intensa, por lo que podría existir un mayor riesgo de recaída. Por tanto, los médicos de atención primaria y los especialistas que trabajan en salud mental deberán prestar especial atención ante esta posibilidad, evaluando la situación y examinando los posibles signos de uso indebido de sustancias en sus pacientes.

\section{Agradecimientos}

Este trabajo ha sido financiado parcialmente por el Gobierno del Principado de Asturias PCTI 2018-2022 IDI/2018/235 con financiación FEDER por el Instituto Sanitario de Investigación del Principado de Asturias (ISPA), el Centro de Investigación Biomédica en Red de Salud Mental (CIBERSAM) y el Ministerio de Economía, Industria y Competitividad.

Agradecer a todos los integrantes del Grupo de Trabajo IPSI-COVID19 del Área de Psiquiatría, Universidad de Oviedo, CIBERSAM, ISPA, SESPA, su esfuerzo e implicación en el mencionado proyecto (nombrados por orden alfabético): Iciar Abad, Clara Alvarez-Vázquez, Ma Teresa Bascarán-Fernández, Julio Bobes, M ${ }^{\mathrm{a}}$ Teresa Bobes-Bascarán, Silvia Casaprima-Suárez, Claudia Fernández-Delgado, Lorena de la Fuente-Tomás, Leticia García-Alvarez, Ainoa García-Fernández, M ${ }^{\mathrm{a}}$ Paz García-Portilla, Leticia González-Blanco, Luis Jiménez-Treviño, Pedro Marina, Clara Martínez Cao, Isabel Menéndez-Miranda, Carlota Moya-Lacasa, Gonzalo Paniagua, Angeles Paredes, Cristina Pedrosa, Almudena Portilla, Leonor Riera, Julia Rodríguez-Revuelta, Pilar A Sáiz, Francesco dal Santo, Elisa Seijo, María Suárez-Alvarez, Mercedes Valtueña-García, Angela Velasco-Iglesias, Paula Zurrón.

\section{Conflicto de intereses}

Los autores declaran que no existe conflicto de interés.

\section{Referencias}

Arango, C. (2020). Lessons learned from the coronavirus health crisis in Madrid, Spain: How COVID-19 has changed our lives in the last two weeks. Biological Psychiatry. Avance de publicación on-line. doi:10.1016/j. biopsych.2020.04.003. 
Bai, Y., Lin, C. C., Lin, C. Y., Chen, J. Y., Chue, C. M. y Chou, P. (2004). Survey of stress reactions among health care workers involved with the SARS outbreak. Psychiatry Service, 55, 1055-1057. doi:10.1176/appi.ps.55.9.1055.

Ben-Zur, H. y Zeidner, M. (2009). Threat to life and risk-taking behaviors: a review of empirical findings and explanatory models. Personality and Social Psychology Review, 13, 109-128. doi:10.1177/1088868308330104.

Boscarino, J. A., Adams, R. E. y Galea, S. (2006). Alcohol use in New York after the terrorist attacks: a study of the effects of psychological trauma on drinking behavior. Addictive Behaviors, 31, 606-621. doi:10.1016/j.addbeh.2005.05.035.

Brooks, S. K., Webster, R. K., Smith, L. E., Woodland, L., Wessely, S., Greenberg, N. y Rubin, G. J. (2020). The psychological impact of quarantine and how to reduce it: rapid review of the evidence. Lancet, 395, 912-920. doi:10.1016/S0140-6736(20)30460-8.

Cai, H. (2020). Sex difference and smoking predisposition in patients with COVID-19. The Lancet Respiratory Medicine, 8, e20. doi:10.1016/S2213-2600(20)30117-X.

Chong, M. Y., Wang, W. C., Hsieh, W. C., Lee, C. Y., Chiu, N. M., Yeh, W. C., . . Chen, C. L. (2004). Psychological impact of severe acute respiratory syndrome on health workers in a tertiary hospital. The British Journal of Psychiatry, 185, 127-133. doi:10.1192/bjp.185.2.127.

Degenhardt, L., Hall, W. y Lynskey, M. (2001). The relationship between cannabis use and other substance use in the general population. Drug and Alcohol Dependence, 64, 319-327.

García-Alvarez, L., de la Fuente-Tomás, L., García-Portilla, M. P., Saiz, P. A., Moya-Lacasa, C., dal Santo, F., ...Bobes, J. (2020) Early impact of the 2019 coronavirus disease (COVID-19) pandemic and lockdown in a large Spanish sample. Psychological Medicine. Manuscrito remitido para publicación.

Gross, G. M., Bastian, L. A., Smith, N. B., Harpaz-Rotem, I. y Hoff, R. (2020). Sex differences in associations between depression and posttraumatic stress disorder symptoms and tobacco use among veterans of recent conflicts. Journal of Womens Health (Larchmt). Avance de publicación on-line. doi:10.1089/jwh.2019.8082.

Japuntich, S. J., Gregor, K., Pineles, S. L., Gradus, J. L., Street, A. E., Prabhala, R. y Rasmusson, A. M. (2016). Deployment stress, tobacco use, and postdeployment posttraumatic stress disorder: Gender differences. Psychological Trauma, 8, 123-126. doi:10.1037/tra0000093.

Jeong, H., Yim, H. W., Song, Y. J., Ki, M., Min, J. A., Cho, J. y Chae, J. H. (2016). Mental health status of people isolated due to Middle East respiratory syndrome. Epidemiology and Health, 38, e2016048. doi:10.4178/epih. e2016048.

Jiménez-Treviño, L., Velasco, A., Rodriguez-Revuelta, J., Abad, I., De la Fuente-Tomás, L., González-Blanco, L.,
... Saiz, P. A. (2019). Factors associated with tobacco consumption in patients with depression. Adicciones, 31, 298-308. doi:10.20882/adicciones.1191.

Ko, C. H., Yen, C. F., Yen, J. Y. y Yang, M. J. (2006). Psychosocial impact among the public of the severe acute respiratory syndrome epidemic in Taiwan. Psychiatry and Clinical Neurosciences, 60, 397-403. doi:10.1111/j.14401819.2006.01522.x.

Lam, M. H., Wing, Y. K., Yu, M. W., Leung, C. M., Ma, R. C., Kong, A. P., . . . Lam, S. P. (2009). Mental morbidities and chronic fatigue in severe acute respiratory syndrome survivors: long-term follow-up. Archives of Internal Medicine, 169, 2142-2147. doi:10.1001/archinternmed.2009.384.

Lebeaut, A., Tran, J. K. y Vujanovic, A. A. (2020). Posttraumatic stress, alcohol use severity, and alcohol use motives among firefighters: The role of anxiety sensitivity. Addictive Behaviors, 106, 106353. doi:10.1016/j.addbeh.2020.106353.

Lee, S. M., Kang, W. S., Cho, A. R., Kim, T. y Park J.K. (2018). Psychological impact of the 2015 MERS outbreak on hospital workers and quarantined hemodialysis patients. Comprehensive Psychiatry, 87, 123-127. doi:10.1016/j.comppsych.2018.10.003.

Lima, C. K. T., Carvalho, P. M. M., Lima, I., Nunes, J., Saraiva, J. S., de Souza, R. I., . . Neto, M. L. R. (2020). The emotional impact of Coronavirus 2019-nCoV (new Coronavirus disease). Psychiatry Research, 287, 112915. doi: 10.1016/j.psychres.2020.112915.

Mak, I. W., Chu, C. M., Pan, P. C., Yiu, M. G. y Chan, V. L. (2009). Long-term psychiatric morbidities among SARS survivors. General Hospital Psychiatry, 31, 318-326. doi:10.1016/j.genhosppsych.2009.03.001.

Maunder, R., Hunter, J., Vincent, L., Bennett, J., Peladeau, N., Leszcz, M., . . . Mazzulli, T. (2003). The immediate psychological and occupational impact of the 2003 SARS outbreak in a teaching hospital. Canadian Medical Association Journal, 168, 1245-1251.

North, C. S., Kawasaki, A., Spitznagel, E. L. y Hong, B. A. (2004). The course of PTSD, major depression, substance abuse, and somatization after a natural disaster. The Journal of Nervous and Mental Disease, 192, 823-829. doi:10.1097/01.nmd.0000146911.52616.22.

Park, S. C. y Park, Y. C. (2020). Mental health care measures in response to the 2019 novel coronavirus outbreak in Korea. Psychiatry Investigation, 17, 85-86. doi:10.30773/ pi.2020.0058.

Pfefferbaum, B. y North, C. S. (2020). Mental health and the Covid-19 pandemic. The New England Journal of Medicine. Avance de publicación on-line. doi:10.1056/NEJMp2008017.

Reger, M. A., Stanley, I. H. y Joiner, T. E. (2020). Suicide mortality and coronavirus disease 2019-A perfect 
storm? JAMA Psychiatry. Avance de publicación on-line. doi:10.1001/jamapsychiatry.2020.1060.

Sánchez Autet, M., Garriga, M., Zamora, F.J., González, I., Usall, J., Tolosa, L., ...Arranz, B. (2018). Screening of alcohol use disorders in psychiatric outpatients: influence of gender, age, and psychiatric diagnosis. Adicciones, 30, 251-263. doi:10.20882/adicciones.885.

Shimizu, S., Aso, K., Noda, T., Ryukei, S., Kochi, Y. y Yamamoto, N. (2000). Natural disasters and alcohol consumption in a cultural context: the Great Hanshin Earthquake in Japan. Addiction, 95, 529-536. doi:10.1046/j.13600443.2000.9545295.x.

Volkow, N. D. (2020). Collision of the COVID-19 and addiction epidemics. Annals of Internal Medicine. Avance de publicación on-line. doi:10.7326/m20-1212.

Wang, C., Pan, R., Wan, X., Tan, Y., Xu, L., Ho, C. S. y Ho, R. C. (2020). Immediate psychological responses and associated factors during the initial stage of the 2019 coronavirus disease (COVID-19) epidemic among the general population in China. International Journal of Environmental Research and Public Health, 17. Avance de publicación on-line. doi:10.3390/ijerph17051729.

Wu, P., Liu, X., Fang, Y., Fan, B., Fuller, C. J., Guan, Z., . . . Litvak, I. J. (2008). Alcohol abuse/dependence symptoms among hospital employees exposed to a SARS outbreak. Alcohol and Alcoholism, 43, 706-712. doi:10.1093/ alcalc/agn073.

Xiang, Y. T., Yang, Y., Li, W., Zhang, L., Zhang, Q., Cheung, T. y Ng, C. H. (2020). Timely mental health care for the 2019 novel coronavirus outbreak is urgently needed. Lancet Psychiatry, 7, 228-229. doi:10.1016/S22150366(20)30046-8.

Yoon, M. K., Kim, S. Y., Ko, H. S. y Lee, M. S.(2016). System effectiveness of detection, brief intervention and refer to treatment for the people with post-traumatic emotional distress by MERS: a case report of community-based proactive intervention in South Korea. International Journal of Mental Health Systems, 10, 51. doi:10.1186/s13033016-0083-5. 